\title{
Scorpion Venom Polypeptide Inhibits Pulmonary Epithelial-mesenchymal Transition in Systemic Sclerosis-interstitial Lung Disease Model Mice by Intervening TGF- $\beta 1 /$ Smad Signaling Pathway
}

\section{Yan Zhang}

The First Affiliated Hospital of Zhejiang Chinese Medical University, Zhejiang Provincial Hospital of Traditional Chinese Medicine

\section{Liping Xu}

The First Affiliated Hospital of Zhejiang Chinese Medical University, Zhejiang Provincial Hospital of Traditional Chinese Medicine

\section{Qiang Chen}

Affiliated Xiaoshan Hospital, Hangzhou Normal University

\section{Tianrong Guan}

The First Affiliated Hospital of Zhejiang Chinese Medical University, Zhejiang Provincial Hospital of

Traditional Chinese Medicine

\section{$\mathrm{Na}$ Lin}

The First Affiliated Hospital of Zhejiang Chinese Medical University, Zhejiang Provincial Hospital of

Traditional Chinese Medicine

\section{Danyang Xu}

The First Affiliated Hospital of Zhejiang Chinese Medical University, Zhejiang Provincial Hospital of

Traditional Chinese Medicine

\section{Lihong Lu}

The First Affiliated Hospital of Zhejiang Chinese Medical University, Zhejiang Provincial Hospital of Traditional Chinese Medicine

\section{Qiaoding Dai}

The First Affiliated Hospital of Zhejiang Chinese Medical University, Zhejiang Provincial Hospital of

Traditional Chinese Medicine

\section{Xinwei Song ( $\nabla$ sxw1055@163.com )}

The First Affiliated Hospital of Zhejiang Chinese Medical University, Zhejiang Provincial Hospital of Traditional Chinese Medicine 
Keywords: Scorpion venom polypeptide, systemic sclerosis, interstitial lung disease, pulmonary fibrosis, epithelial-mesenchymal transition, TGF- $\beta 1 /$ Smad signaling pathway

Posted Date: February 21st, 2022

DOI: https://doi.org/10.21203/rs.3.rs-1238400/v1

License: (1) This work is licensed under a Creative Commons Attribution 4.0 International License. Read Full License

Version of Record: A version of this preprint was published at Evidence-Based Complementary and Alternative Medicine on April 13th, 2022. See the published version at https://doi.org/10.1155/2022/6557486. 


\section{Abstract \\ Objective}

Systemic sclerosis (SSc) is a rare autoimmune connective tissue disease, and interstitial lung disease (ILD) is an important complication of SSc. The aim of this study was to investigate the effect of polypeptide extract of scorpion venom (PESV) on alleviating pulmonary epithelial-mesenchymal transition (EMT) in SSc-ILD mice and its possible mechanism.

\section{Methods}

C57/BL6 mice were injected with bleomycin to establish a SSc-ILD model. Different concentrations of PESV solution were administered, and dexamethasone was used as a positive control. Then the lung tissues of mice in each group were removed, and the pathological changes of mice in each group were observed using HE staining and Masson staining. TGF- $\beta 1$ expression levels were detected by immunohistochemical. The expression of EMT-related proteins E-cadherin, collagenl, vimentin, $\mathrm{N}$ cadherin, and a-SMA was detected by Western blot, and the expression of TGF- $\beta 1 /$ Smad pathway-related proteins was also detected. The contents of inflammatory cytokines IL-6, TNF- $\alpha$ and TGF- $\beta 1$ in serum and BALF were also measured by ELISA.

\section{Results}

Pathological analysis showed that PESV alleviated the SSc-ILD induced pulmonary inflammation and fibrosis. The results showed that after PESV treatment, the contents of inflammatory cytokines IL- 6 and TNF-a were significantly lower than those in the model group. PESV could increase the expression of epithelial marker E-cadherin and reduce the expression of interstitial markers collagenl, vimentin, $\mathrm{N}$ cadherin, and a-SMA. PESV can reduce the expression level of TGF- $\beta 1 /$ Smad pathway-related proteins .

\section{Conclusion}

PESV can inhibit the EMT and relevant TGF- $\beta 1 /$ Smad signaling pathway, to alleviate pulmonary inflammation and fibrosis, thus to play a protective effect against SSc-ILD.

\section{Background}

Systemic sclerosis (SSc) is an immune-mediated rheumatic disease that is characterised by fibrosis of the skin and internal organs and vasculopathy. ${ }^{1}$ Despite evidence of improved survival, SSc has a high mortality greater than any other rheumatic disease. ${ }^{2,3}$ Skin fibrosis is the most common manifestation of SSc, and fibrosis of internal organs leads to organ damage and poor prognosis. 
Up to $80 \%$ of patients with SSc have interstitial lung disease (ILD) on high-resolution computed tomography (HRCT) scan, and $30-40 \%$ of patients with SSc have clinically significant ILD, ${ }^{4}$ SScassociated ILD is the leading cause of disease-related mortality. The basic pathological changes in ILD are interstitial fibrosis, diffuse parenchymal, and alveolar inflammation. At present, there is no effective drug that can cure the SSc-ILD, and mainly using glucocorticoid combined with immunosuppressants such as cyclophosphamide, metecophenol ester, azathioprine, etc. ${ }^{5}$ The pathogenesis of SSc-ILD are not completely unraveled, several studies have suggested that Epithelial-mesenchymal transition (EMT) may take a place in the skin of patients with SSc and may have a role in the pathogenesis of ILD. ${ }^{6,7}$ Inhibition of EMT may be represent a novel therapeutic of SSC-ILD.

Transforming growth factor $\beta$ (TGF- $\beta$ ) is a multifunctional cytokine involved in processes such as cell wound repair and fibrosis, of which TGF- $\beta 1$ is the most ample isoform. ${ }^{8}$ TGF- $\beta 1$ is a profibrotic factor, and the TGF- $\beta 1 /$ Smad signaling pathway it is involved is also closely related to the fibrosis and organ sclerosis. ${ }^{9}$ Kim S et al reported that the TGF- $\beta 1 /$ Smad signaling pathway plays an important role in the pathogenesis of pulmonary fibrosis. ${ }^{10}$ In addition, study also suggested that TGF- $\beta 1$ can induce EMT changes in cultured respiratory epithelial cells and that the TGF- $\beta 1 / \mathrm{Smad}$ pathway may regulate airway EMT and physiological obstruction through fibrosis ${ }^{11}$.

Scorpion is the dried whole of Buthus martensii Karsch, it is used as a traditional Chinese medicinal herb with anti-tumor, sedative and analgesic, and anticonvulsant effects. It is commonly used in the treatment of diseases such as malignant tumors, convulsions, and rheumatism. ${ }^{12}$ The chemical constituents in scorpion mainly contain the polypeptide extract of scorpion venom (PESV), water-soluble components, and fat-soluble components. ${ }^{13}$ Previous studies have found that the PESV is able to reduce the expression of TGF- $\beta 1 .{ }^{14}$ PESV can effectively restore lung cancer immune escape by reducing the levels of TGF- $\beta$, IL-10, and VEGF. ${ }^{15}$ Compared to the increasing studies of PESV in lung cancer, study about PESV on SSC-ILD is nearly none.

PESV have a regulatory effect on TGF- $\beta 1$ and have also been used in the treatment of some lung diseases. In addition, the TGF- $\beta 1 /$ Smad signaling pathway, which is involved by TGF- $\beta 1$, is closely related to the treatment of pulmonary fibrosis. Hence, we hypothesize that PESV could improve the pulmonary fibrosis by regulating TGF- $\beta 1 /$ Smad and pulmonary EMT, thus to alleviate SSc-ILD. In this study, we established a mouse model of SSc-ILD and intervened with different concentrations of PESV, with the aims to study the effect of PESV on the symptoms of SSc-ILD and its mechanism.

\section{Material And Methods}

\subsection{Grouping and establishment of experimental animals}

Forty-eight healthy adult female SPF grade C57/BL6 mice aged 6-8 weeks with the body weight of 20-22 $\mathrm{g}$ were randomly divided into six groups: normal control group (NC group), bleomycin-induced SSc-ILD model group (BLM group), low-dose PESV intervention group (PESV-L group), medium-dose PESV 
intervention group (PESV-M group), high-dose PESV intervention group (PESV-H group), and dexamethasone intervention group (DXM group), with eight mice in each group. The study was carried out in accordance with the Declaration of Helsinki. This animal experimental program has been reviewed by the Experimental Animal Management and Ethics Committee of Zhejiang University of Traditional Chinese Medicine and conforms to the principles of animal protection, animal welfare and ethics, as well as the relevant provisions of China's National Experimental Animal Welfare Ethics. Animal Experiment Ethics Approval Number: IACUC-20210524-03.

SSc-ILD animal modelling in this study is based on reports from Aso $Y^{16}$ and Yamamoto $T^{17,18}$ et al. After removing the $2.0 \times 2.0 \mathrm{~cm}$ hair from the center of the back in each group mice with $10 \%$ sodium sulfide solution, mice in the NC group were subcutaneously injected with $0.1 \mathrm{ml}$ of normal saline. Except for the $\mathrm{NC}$ group, the rest groups were subcutaneously injected with $0.1 \mathrm{ml}$ bleomycin solution $(1 \mathrm{mg} / \mathrm{ml}$, Hanhui Pharmaceutical Co., Ltd., 2006741) once a day for 4 weeks to establish a mouse SSc-ILD model. In addition, PESV-L group, PESV-M group, PESV-H group, and DXM group were intragastrically administered with $5 \mathrm{mg} / \mathrm{kg}$ PESV solution (Zhengzhou Licheng Biotechnology Co., Ltd.), $10 \mathrm{mg} / \mathrm{kg}$ PESV solution, 20 $\mathrm{mg} / \mathrm{kg}$ PESV solution, and $0.15 \mathrm{mg} / \mathrm{kg}$ dexamethasone sodium phosphate injection (Suicheng Pharmaceutical Solution Co., Ltd.) for 4 weeks from the day of modeling, respectively. ${ }^{19}$ The activity status, mental status, respiration, fur change, food intake, water intake and other general conditions of mice in each group were observed during the experiment.

\subsection{Bronchoalveolar lavage fluid (BALF), lung and serum collection}

After 4 weeks of administration, the mice in each group were sacrificed, and blood samples were collected from the mice in each group (eyeball removal method) and placed in sterilized EP tubes overnight $\left(4^{\circ} \mathrm{C}\right)$. Blood was centrifuged after clotting $\left(4^{\circ} \mathrm{C}, 10 \mathrm{~min}, 2000 \mathrm{rpm}\right)$. The collected supernatant was stored at $-80^{\circ} \mathrm{C}$.

The skin of the anterior cervical region of the mice was cut open. A red intravenous catheter was placed at the distal end of the trachea and ligated and fixed. One milliliter of normal saline was then infused into the lung tissue. After the lung was significantly filled, it remained for 10 seconds for pumping back, which was repeated three times. Finally, the collected BALF was centrifuged $\left(4^{\circ} \mathrm{C}, 800 \mathrm{rpm}, 5 \mathrm{~min}\right)$ and stored at $-80^{\circ} \mathrm{C}$ until use. After that, lung tissues form the each group mice were dissected, a portion was fixed in $10 \%$ neutral formaldehyde, another portion was stored at $-80^{\circ} \mathrm{C}$.

\subsection{HE staining}

Lung tissue samples (10\% neutral formaldehyde (Fuzhou Feijing Biotechnology Co., Ltd.)) from mice in each group were fixed and cut into $4-\mu m$-thick paraffin sections. Deparaffinize to water. Hematoxylin staining solution was added, followed by differentiation ( $0.5 \%$ hydrochloric acid in alcohol, $10 \mathrm{~s}$ ) and reversion to blue ( $1 \%$ diluted ammonia, $90 \mathrm{~s}$ ). Eosin staining solution (60 s) was then added for staining, and the results were observed under a microscope after fixation with neutral resin. 


\subsection{Masson's staining}

The lung tissues of mice in each group were deparaffinized to water after sectioning, stained with iron hematoxylin staining solution (10 $\mathrm{min}$ ), refluxed with blue solution ( $5 \mathrm{~min}$ ), and finally stained with red staining solution (15 min). After differentiation (1\% acetic acid, $10 \mathrm{~s}$ ) they were fixed with neutral gum. Observations were performed and assessed microscopically.

\subsection{Immunohistochemical staining}

Lung tissue sections from mice in each group were deparaffinized to water and subjected to antigen heat retrieval. Primary TGF- $\beta 1$ antibody (ImmunoWay Biotechnology Company, USA) was added followed by overnight incubation at $4{ }^{\circ} \mathrm{C}$. Secondary antibodies were added the following day and incubated for half an hour at $37^{\circ} \mathrm{C}$. Then DAB chromogenic solution was added, and the staining results were observed microscopically at $200 \times$ field after counterstaining with hematoxylin.

\subsection{Western blot}

The lung tissue samples of mice in each group were crushed and lysed, centrifuged and the total protein concentration was measured with a BCA kit, followed by SDS-PAGE electrophoresis, and then transferred to a PVDF membrane. At the end of the transfer membrane, blocking was performed at room temperature for $1 \mathrm{~h}$ ( $5 \%$ non-fat dry milk), and then the corresponding primary antibodies (E-cadherin Antibody (Affinity, AF0131), Collagen I Antibody (Affinity, AF7001), Vimentin Antibody (Affinity, AF7013), N-cadherin

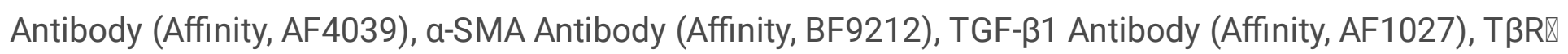
Antibody (Affinity, DF7309), T $\beta R \bigotimes$ Antibody (Affinity, DF13307), Phospho-Smad2 (Ser250) Antibody (Affinity, AF3450), Smad2 Antibody (Affinity, AF6449), Phospho-Smad3 (Ser423+Ser425) Antibody (Affinity, AF8315), Smad3 Antibody (Affinity, AF6362), Smad7 Antibody (Affinity, AF5147)) were incubated at $4{ }^{\circ} \mathrm{C}$ overnight. After incubation of HRP-labeled secondary antibody at room temperature for $1 \mathrm{~h}$, the developing solution was added for luminescence and development and fixation. After scanning the film, the gray values of each band were determined with image analysis software, and the ratio with the internal reference $\beta$-actin was used as the basis for semi-quantitative analysis.

\subsection{ELISA test}

Take standby BALF and serum, place them at room temperature for 20 min, add distilled water to dilute 20 times concentrated washing solution into original washing solution. The corresponding inflammatory factor concentrations in BALF were measured according to the manufacturer's instructions of the ELISA kit. The tested ELISA kits include Mouse IL6 (Interleukin 6) ELISA Kit (ELK1157, ELK Biotechnology), Mouse TGF $\beta 1$ (Transforming Growth Factor Beta 1) ELISA Kit (ELK1186, ELK Biotechnology), and Mouse TNFa (Tumor Necrosis Factor Alpha) ELISA Kit (ELK1387, ELK Biotechnology).

\subsection{Statistical analysis}


All data from this experiment were analyzed and processed using SPSS 16.0 as well as GraphpadPrism7 software. All data were expressed as mean \pm standard deviation $(\bar{x} \pm s), P<0.05$ was considered statistically significant. One-way-ANOAY analysis of variance was used for multiple groups of measurement data, and SNK test was used for comparison. Kruskal-WallisH test was used for unequal or non-normal variance.

\section{Results}

\subsection{General condition of the mice}

Mice in the NC group showed normal activity status, good mental status, shiny coat color, soft skin, and a normal diet. Mice in the BLM group had symptoms consistent with SSc relative to mice in the NC group. These include not much activity, apathy, most curled up in corners, tarnished coat color, hardened skin epidermis, and reduced eating. While these symptoms were alleviated to varying degrees in the mice of the drug-treated groups.

\subsection{Pathological changes of lung tissues}

The lung tissue in NC group was clear and the alveolar structure was intact. In contrast, the BLM group clearly had a large number of fibrocyte and inflammatory cell infiltrates, increased lung macrophages, significantly thicker septa between alveoli, and severely destroyed alveolar structures. The degree of lung tissue lesions in PESV-H group, PESV-M group, PESV-M group and DXM group was significantly alleviated compared with the BLM group. The pathological changes were improved to different extents after treatment with different concentrations of PESV in a dose-dependent manner (Figure. 1).

\subsection{Fibrotic changes in lung tissue}

As can be seen from Figure 2, the masson staining of lung tissues showed that the alveolar structure of the NC group was normal without significant fibrotic changes. In the BLM group, the alveolar structure was severely destroyed, and a large amount of fibrous hyperplasia stained with blue was observed, showing the collagen bundle formation of lung. The results of the PESV-L, PESV-M, PESV-H, and DXM groups showed that although fibrous hyperplasia was still observed, it was significantly milder than that of the BLM group.

\subsection{Content of inflammatory cytokines IL-6, TNF- $\alpha$ and TGF- $\beta 1$ in serum and BALF}

The contents of IL-6, TNF- $\alpha$ and TGF- $\beta 1$ in serum and BALF were detected by ELISA. Results are shown in Figure 3. It can be seen that the change trends for the contents of these inflammatory cytokines in serum and BALF were coincident. Compared with the NC group, the contents of inflammatory factors TGF- $\beta 1$, IL6 , and TNF-a in serum and BALF were significantly increased $(P<0.05$ or $P<0.01)$. Compared with BLM group, the contents of these factors were significantly decreased after treated with PESV and positive drug (dexamethasone) $(P<0.05$ or $P<0.01)$. The inflammatory factors changes were improved to different extents after treatment with different concentrations of PESV in a dose-dependent manner. 


\subsection{Expression of EMT related proteins in lung tissue}

The results of Western blot showed that the protein expression of epithelial marker as E-cadherin in the lung tissues of mice in the BLM group was significantly lower than that in the NC group $(P<0.01)$, while the protein expression levels of mesenchymal markers as collagen I, vimentin, N-cadherin, and a-SMA were significantly higher than those in the NC group $(P<0.01)$. After treatment with different concentrations of PESV and DXM, the results were reversed compared with BLM. (Figure 4).

\subsection{Expression of TGF- $\beta 1$ in lung tissue}

It can be seen from Figure 5 that the expression level of TGF- $\beta 1$ protein in BLM group was significantly higher than that in NC group, with obviously more brown stained cells. While the expression level of TGF$\beta 1$ protein in PESV-L group, PESV-M group, PESV-H group, and DXM group was significantly lower than that in BLM group.

\subsection{Expression of TGF- $\beta 1 /$ Smad signaling pathway related proteins in lung tissue}

As shown in Figure 6, the expression levels of TGF- $\beta 1, T \beta R I, T \beta R I l, p-S m a d 2$, and p-Smad3 proteins in the lung tissues of the BLM group mice were significantly higher than those in the NC group $(P<0.01)$, and the expression levels of Smad7 protein were significantly lower $(P<0.01)$. After treatment with different concentrations of PESV and DXM, the results were reversed compared with the BLM group.

\section{Discussion}

SSc is a rheumatic immune disease characterized by skin and organ fibrosis. Studies concluded that the pathogenesis of SSc (including skin, lung and other tissue fibrosis) was associated with abnormal function of endothelial cell damage and activation of collagen, which stimulates fibroblast synthesis, which in turn leads to fibrosis of blood vessel walls and tissues. ${ }^{20}$ The key mechanism of SSc-ILD pathogenesis is persistent lung damage caused by alveoli macrophage activation, inducing the release of a variety of fibrosis-promoting cytokines increased, prompting the conversion of pulmonary epithelial cells to interstitial cells (EMT), characterized by epithelial cell markers E-cadherin, ZO-1 inactivation, mesenchymal cell markers collagen I, vimentin, N-cadherin, and a-SMA and other activated expression, which eventually led to the formation of pulmonary fibrosis. ${ }^{21}$

At present, various cytokine level abnormalities have been found in serum and affected lung tissue in SSc-ILD patients, such as TGF- $\beta 1$, TNF- $\alpha$, IL-6, etc., and these cytokines are thought to promote fibroblast activation and EMT, which play a key role in the occurrence of SSc-ILD inflammation and fibrosis. ${ }^{22}$ In the cytokine network involved in SSc-ILD, TGF- $\beta$ 1 is the most in-depth and important cytokine in the current study, which regulates the expression of matrix metalloproteinases (MMP) and extracellular matrix (ECM) by activating EMT-related transcription factors to promote epithelial apoptosis, epithelial cell migration, and EMT. ${ }^{23}$ It has been shown that PESV, an active ingredient of Buthus martensii Karsch, can effectively improve the expression of E-cadherin, ${ }^{24}$ a epithelial marker in leukemia mice, and can interfere with the 
growth and metastasis of Lewis lung carcinomas in mice by inhibiting MMP-9. ${ }^{25}$ PESV also has a significant inhibitory effect on the EMT process of liver cancer cells, which can reduce the invasiveness of liver cancer cells and inhibit further metastasis of liver cancer. ${ }^{26}$ The above research suggests that PESV may have the effect of inhibiting EMT and reducing tissue fibrosis.

In our study, the SSc-ILD model of mice was established by subcutaneous injection of bolemycin, and the results showed skin hardening in the back injection area of BLM group mice, and the results of pulmonary tissue HE staining and Masson staining showed alveoli inflammation, fibrosis and hyperplantation, respectively, consistent with the results of Wolflin $L$ et al., ${ }^{27}$ indicating the success of SSc-ILD model construction. Our further results suggest that PESV can significantly improve typical symptoms and pathological changes in the lungs of SSc-ILD model mice. The contents of TGF- $\beta 1$, TNF- $a$ and IL- 6 in mice and BALF were detected by ELISA method, and the results showed that compared with NC group, the content of inflammatory factors TGF- $\beta 1$, TNF- $\alpha$ and IL- 6 in model group mice was significantly increased. Compared with BLM group, after treatment of different concentrations of PESV, the above-mentioned inflammatory factor content decreased and dose dependence was shown, suggesting that PESV played a role in improving inflammation of the whole body and lungs of SSc-ILD model mice. In addition, our study found that PESV has an active effect on epithelial cell marker Ecadherin, and inhibits mesenchymal cell markers collagen I, Vimentin, N-cadherin, a-SMA, suggesting that PESV inhibits the EMT process in mice with SSc-ILD model.

TGF- $\beta 1$ is one of the common factors that trigger pulmonary fibrosis, and TGF- $\beta 1$ overexpression causes pulmonary fibrosis, while TGF- $\beta 1$ inhibitors can reduce the degree of pulmonary fibrosis. ${ }^{28-31}$ Numerous studies have confirmed that TGF- $\beta 1$ can promote tissue fibrosis by inducing EMT. ${ }^{32,33}$ EMT is a critically important event in tissue fibrosis, it is characterized by the deactivation of the epithelial cell markers, including E-cadherin, ZO-1, and the activated expression of mesenchymal cell markers, such as collagen I, N-cadherin, vimentin, a-SMA, Fibronectin. ${ }^{34,35}$ Further, we detected significantly higher levels of TGF- $\beta 1$ protein expression in BLM group lung tissue than in NC group and PESV intervention by examining the expression of TGF- $\beta 1$ and EMT-related proteins in mice in each experimental group, the expression level of epithelial cell marker E-cadherin protein in the lung tissue of mice in BLM group was significantly lower than that of NC group and PESV group, mesenchymal cell marker collagen I, Vimentin, N-cadherin, a-SMA protein expression levels were significantly higher than those in the NC group and the PESV group. It is shown that TGF- $\beta 1$ expression is positively correlated with EMT process, and it is further shown that PESV can inhibit TGF- $\beta 1$ expression and EMT process.

Additionally, the induction of EMT by TGF- $\beta 1$ is closely related to Smad2/3, Smad7. ${ }^{36,37}$ Lee SH et al reported that TGF- $\beta 1$ inhibited fibrosis by inducing the phosphorylation of Smad2/3. ${ }^{38}$ Lee CM et al reported that the mediating effect of TGF- $\beta 1$ on the fibrotic response is closely related to the inhibition of the expression of the downstream factor Smad7. ${ }^{39}$ We experimentally found that the expression of TGF$\beta 1 / \mathrm{Smad} 2 / 3$ pathway-related proteins in SSc-ILD model mice was significantly higher than that in the control group, and there was a significant decrease in pathway-related proteins after PESV treatment. 
This indicated that the inhibitory effect of PESV on EMT in SSc-ILD model mice may be related to the TGF- $\beta 1 /$ Smad pathway.

In previous studies on PESV, there are few mechanisms related to the TGF- $\beta 1$ pathway, but PESV has a regulatory effect on TGF- $\beta 1$ reflected in a few literatures.15,16 In our study, PESV was found to have a significant inhibitory effect on the expression of TGF- $\beta 1$ and its receptor through the detection of TGF- $\beta 1$ and its receptor. Combined with the promoting effect of TGF- $\beta 1$ on EMT in SSC-ILD model mice, it is indicated that PESV may achieve the inhibition of EMT by regulating TGF- $\beta 1$ pathway, thereby alleviating pulmonary inflammation and interstitial fibrosis in mice.

\section{Conclusion}

In conclusion, this study investigated the therapeutic potential of PESV to treat SSc-ILD. Studies demonstrated that PESV inhibited the SSc-ILD induced EMT, to alleviate pulmonary inflammation and interstitial fibrosis in model mice, and this effect was acted through the intervention of the TGF- $\beta 1 /$ Smad signaling pathway. These results suggest that PESV may be used as potential drugs for the treatment of SSc-ILD. However, further in depth studies are need to confirm the clear role of PESV in SSc-ILD.

\section{Abbreviations}

SSC: Systemic sclerosis

ILD: Interstitial lung disease

HRCT: High-resolution computed tomography

EMT: Epithelial-mesenchymal transition

TGF- $\beta$ : Transforming growth factor $\beta$

PESV: Polypeptide extract of scorpion venom

NC: Normal control

BLM: Bleomycin

DXM: Dexamethasone

MMP: Matrix metalloproteinases

ECM: Extracellular matrix

\section{Declarations}




\section{Ethics approval and consent to participate}

This animal experiment was completed with the support of the Animal Experimental Center of Zhejiang University of Traditional Chinese Medicine, Number of Animal Use Permit: SYXK (Zhe) 2021-0012. This animal experimental program has been reviewed by the Experimental Animal Management and Ethics Committee of Zhejiang University of Traditional Chinese Medicine and conforms to the principles of animal protection, animal welfare and ethics, as well as the relevant provisions of China's National Experimental Animal Welfare Ethics. Animal Experiment Ethics Approval Number: IACUC-20210524-03. We carried out these experiments in compliance with the ARRIVE guidelines (https://arriveguidelines.org).

\section{Availability of data and materials}

All data generated or analyzed during this study are included in this published article and its important information files. All data can be downloaded through this Digital Object Identifier (DOI):

https://doi.org/10.6084/m9.figshare.19145912. The original blot can be downloaded through this DOI: https://doi.org/10.6084/m9.figshare.19145801.

\section{Consent for publication}

Not applicable.

\section{Availability of data and material}

The datasets used and/or analyzed during the current study are available from the corresponding author on reasonable request.

\section{Competing interests}

There are no competing interests to declare.

\section{Funding}

The work was supported by the National Famous Old Chinese Medicine Experts SONG Xinwei Inheritance Studio Project. (G.TCM.R.J.H.[2018]134)

\section{Authors' contributions}

Y.Z and L.P.X wrote and revised the manuscript (the authors contribute equally), Q.C and T.R.G collected experimental data, N.L and D.Y.X summarized and analyzed experimental data, L.H.L and Q.D.D made experimental charts, and X.W.S drafted and revised the manuscript. All authors reviewed the manuscript.

\section{Acknowledgements}

Not applicable. 


\section{References}

1. Denton CP, Khanna D. Systemic sclerosis. Lancet. 2017;390(10103):1685-1699.

2. van den Hoogen F, Khanna D, Fransen J, Johnson SR, Baron M, Tyndall A, et al. 2013 classifcation criteria for systemic sclerosis: an American College of Rheumatology/European League against Rheumatism collaborative initiative. Arthritis Rheum. 2013; 65(11):2737-2747.

3. Ndosi M, Alcacer-Pitarch B, Allanore Y, Del Galdo F, Frerix M, García-Díaz S, et al. Common measure of quality of life for people with systemic sclerosis across seven European countries: a crosssectional study. Ann Rheum Dis. 2018;77(7):1032-1038.

4. Perelas A, Silver RM, Arrossi AV, Highland KB. Systemic sclerosis-associated interstitial lung disease. Lancet Respir Med. 2020;8(3):304-320.

5. Cottin V, Brown KK. Interstitial lung disease associated with systemic sclerosis (SSc-ILD). Respir Res. 2019;20(1):13.

6. Nikitorowicz-Buniak J, Denton CP, Abraham D, Stratton R. Partially Evoked Epithelial-Mesenchymal Transition (EMT) Is Associated with Increased TGF $\beta$ Signaling within Lesional Scleroderma Skin. PLoS One. 2015;10(7):e0134092.

7. Marmai C, Sutherland RE, Kim KK, Dolganov GM, Fang X, Kim SS, et al. Alveolar epithelial cells express mesenchymal proteins in patients with idiopathic pulmonary fibrosis. Am J Physiol Lung Cell Mol Physiol. 2011;301(1): L71-8.

8. Xu J, Lamouille S, Derynck R. TGF-beta-induced epithelial to mesenchymal transition. Cell Res. 009; 19(2):156-72.

9. Dantas AT, Gonçalves SM, de Almeida AR, Gonçalves RS, Sampaio MC, Vilar KM, et al. Reassessing the Role of the Active TGF- $\beta 1$ as a Biomarker in Systemic Sclerosis: Association of Serum Levels with Clinical Manifestations. Dis Markers. 2016;2016:6064830.

10. Kim S, Han JH, Kim S, Lee H, Kim JR, Lim JH, et al. p90RSK Inhibition Ameliorates TGF- $\beta 1$ Signaling and Pulmonary Fibrosis by Inhibiting Smad3 Transcriptional Activity. Cell Physiol Biochem. 2020;54:195-210.

11. Mahmood MQ, Reid D, Ward C, Muller HK, Knight DA, Sohal SS, et al. Transforming growth factor (TGF) $\beta 1$ and Smad signalling pathways: A likely key to EMT-associated COPD pathogenesis. Respirology. 2017;22(1):133-140.

12. Amr ZS, Abu Baker MA, Al-Saraireh M, Warrell DA. Scorpions and scorpion sting envenoming (scorpionism) in the Arab Countries of the Middle East. Toxicon. 2021;191:83-103.

13. Ding J, Chua PJ, Bay BH, Gopalakrishnakone P. Scorpion venoms as a potential source of novel cancer therapeutic compounds. Exp Biol Med (Maywood). 2014;239(4):387-393.

14. Rincón-Cortés CA, Reyes-Montaño EA, Vega-Castro NA. [Partial purification of peptides present in the Tityus macrochirus (Buthidae) scorpion venom and preliminary assessment of their cytotoxicity]. Biomedica. 2017;37(2):238-249. 
15. Xu L, Zhang W, Wang Z, Jia Q, Zhang YY, Jiang GS. [Effect of polypeptide extract from scorpion venom (PESV) on immune escape of Lewis lung carcinomas]. Zhongguo Zhong Yao Za Zhi, 2010, 35(17): 2324-2327.

16. Aso Y, Yoneda K, Kikkawa Y. Morphologic and biochemical study of pulmonary changes induced by bleomycin in mice. Lab Invest. 1976;35(6):558-68.

17. Yamamoto T, Takagawa S, Katayama I, Yamazaki K, Hamazaki Y, Shinkai H, Nishioka K. Animal model of sclerotic skin. I: Local injections of bleomycin induce sclerotic skin mimicking scleroderma. J Invest Dermatol. 1999;112(4):456-62.

18. Yamamoto T, Kuroda M, Nishioka K. Animal model of sclerotic skin. III: Histopathological comparison of bleomycin-induced scleroderma in various mice strains. Arch Dermatol Res. 2000;292(11): 535-41.

19. Díaz-García A, Pimentel González G, Basaco Bernabeu T, Rodríguez Aurrecochea JC, Rodríguez Sánchez H, Sánchez Monzón I, et al. Pharmacokinetics and Biodistribution of Rhopalurus junceus Scorpion Venom in Tumor-Bearing Mice after Intravenous and Oral Administration. Iran Biomed J. 2019;23(4):287-296.

20. Varga J, Abraham D. Systemic sclerosis: a prototypic multisystem fibrotic disorder. J Clin Invest. 2007;117(3):557-67.

21. Herzog EL, Mathur A, Tager AM, Feghali-Bostwick C, Schneider F, Varga J. Review: interstitial lung disease associated with systemic sclerosis and idiopathic pulmonary fibrosis: how similar and distinct? Arthritis Rheumatol. 2014;66(8):1967-78.

22. Khanna D, Tashkin DP, Denton CP, Renzoni EA, Desai SR, Varga J. Etiology, Risk Factors, and Biomarkers in Systemic Sclerosis with Interstitial Lung Disease. Am J Respir Crit Care Med. 2020;201(6):650-660.

23. Kage H, Borok Z. EMT and interstitial lung disease: a mysterious relationship. Curr Opin Pulm Med. 2012;18(5):517-23.

24. Yang $W H$, Yang XD, Shi ZX. [Scorpion polypeptides interfere with leukemia cell immersion effects and molecular mechanisms]. Tianjin Zhong Yi Yao. 2010;27(1):15.

25. Xu L, Zhang WD, Wang ZP. [Inhibitive effect of polypeptide extract from scorpion venom(PESV)on the growth and metastasis of Lewis lung carcinomas]. Journal of Shandong University (Health Sciences). 2010;48(1):82-93.

26. Yan YQ, Xie J, Wang JF, Shi ZF, Zhang X, Du YP, et al. Scorpion inhibits epithelial-mesenchymal transition and metastasis of hepatocellular carcinoma. Exp Biol Med (Maywood). 2018;243(7):645654.

27. Wollin L, Trinh-Minh T, Zhang Y, Distler JHW. The effect of nintedanib versus mycophenolate mofetil in the Fra2 mouse model of systemic sclerosis-associated interstitial lung disease. Clin Exp Rheumatol. 2021;131(4):134-141.

28. Kang HR, Cho SJ, Lee CG, Homer RJ, Elias JA. Transforming growth factor (TGF)-beta1 stimulates pulmonary fibrosis and inflammation via a Bax-dependent, bid-activated pathway that involves 
matrix metalloproteinase-12. J Biol Chem. 2007;282(10):7723-7732.

29. Tarantal AF, Chen H, Shi TT, Lu CH, Fang AB, Buckley S, et al. Overexpression of transforming growth factor-beta 1 in fetal monkey lung results in prenatal pulmonary fibrosis. Eur Respir J. 2010;36(4):907-914.

30. Wang Y, Kuai Q, Gao F, Wang Y, He M, Zhou H, et al. Overexpression of TIM-3 in Macrophages Aggravates Pathogenesis of Pulmonary Fibrosis in Mice. Am J Respir Cell Mol Biol. 2019;61(6):727736.

31. Boutanquoi PM, Burgy O, Beltramo G, Bellaye PS, Dondaine L, Marcion G, et al. TRIM33 prevents pulmonary fibrosis by impairing TGF- $\beta 1$ signalling. Eur Respir J. 2020;55(6):1901346.

32. Wei JJ, Tang L, Chen LL, Xie ZH, Ren Y, Qi HG, et al. Mesenchymal Stem Cells Attenuates TGF- $\beta 1$ Induced EMT by Increasing HGF Expression in HK-2 Cells. Iran J Public Health. 2021;50(5):908-918.

33. Zhang Q, Chang $X$, Wang $H$, et al. TGF- $\beta 1$ mediated Smad signaling pathway and EMT in hepatic fibrosis induced by Nano NiO in vivo and in vitro. Environ Toxicol, 2020, 35(4): 419-429.

34. Zhang Q, Chang X, Wang H, Liu Y, Wang X, Wu M, et al. IL-17A Promotes Initiation and Development of Intestinal Fibrosis Through EMT. Dig Dis Sci. 2018;63(11):2898-2909.

35. Liu H, Ren SY, Qu Y, Liu C, Zhang Y, Li XQ, et al. MiR-194-5p inhibited metastasis and EMT of nephroblastoma cells through targeting Crk. Kaohsiung J Med Sci. 2020;36(4):265-273.

36. Liu H, He Y, Jiang Z, Shen S, Mei J, Tang M. Prodigiosin Alleviates Pulmonary Fibrosis Through Inhibiting miRNA-410 and TGF- $\beta 1 /$ ADAMTS-1 Signaling Pathway. Cell Physiol Biochem. 2018;49:501-511.

37. Froese AR, Shimbori C, Bellaye PS, Inman M, Obex S, Fatima S, et al. Stretch-induced Activation of Transforming Growth Factor- $\beta 1$ in Pulmonary Fibrosis. Am J Respir Crit Care Med. 2016;194:84-96.

38. Lee SH, Shin JH, Shin MH, Kim YS, Chung KS, Song JH, et al. The Effects of Retinoic Acid and MAPK Inhibitors on Phosphorylation of Smad2/3 Induced by Transforming Growth Factor $\beta 1$. Tuberc Respir Dis (Seoul). 2019;82(1):42-52.

39. Lee $\mathrm{CM}, \mathrm{He} \mathrm{CH}$, Park JW, Lee JH, Kamle S, Ma B, et al. Chitinase 1 regulates pulmonary fibrosis by modulating TGF- $\beta$ /SMAD7 pathway via TGFBRAP1 and FOXO3. Life Sci Alliance. 2019;2(3):e201900350.

\section{Figures}




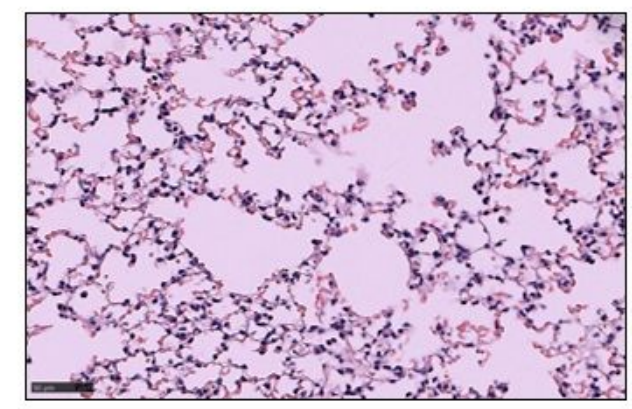

$\mathrm{NC}$

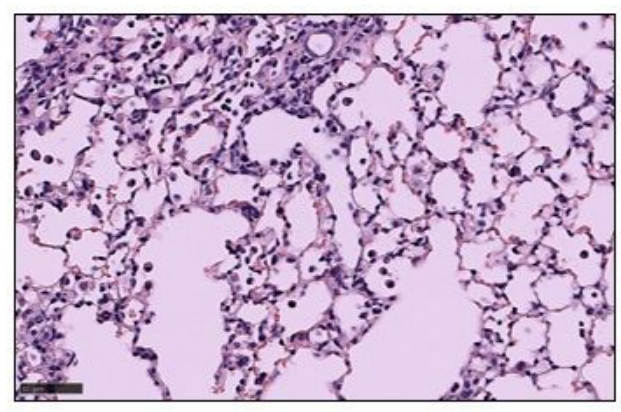

PESV-M

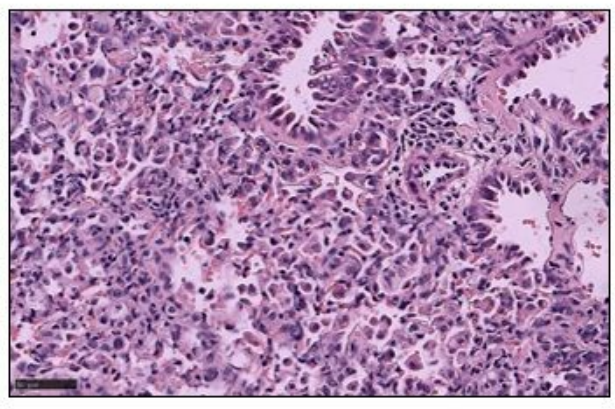

BLM

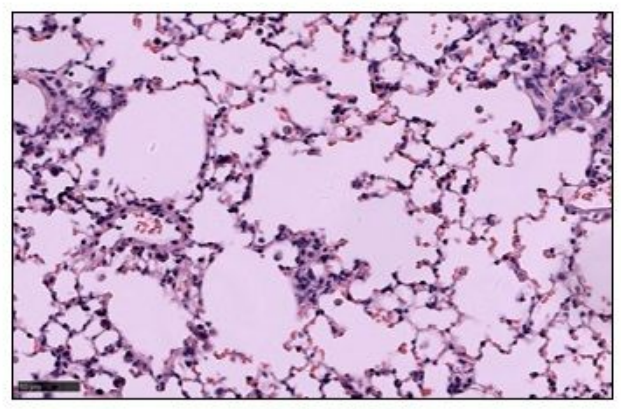

PESV-H

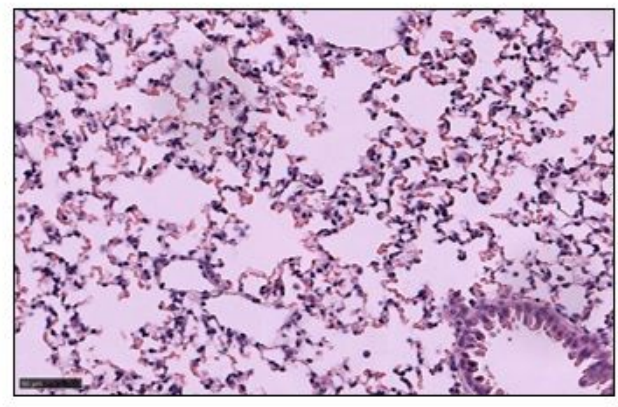

PESV-L

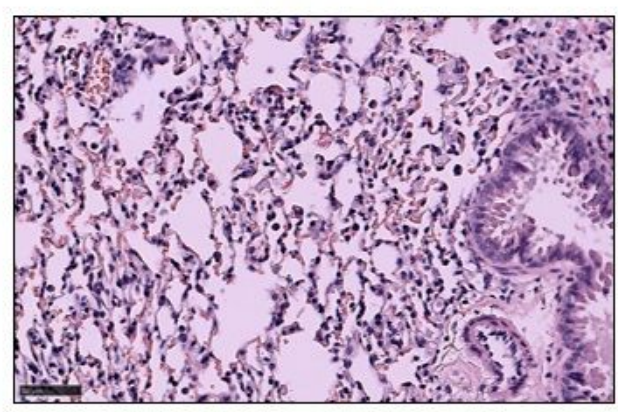

DXM

\section{Figure 1}

Pathological changes of lung tissue in mice of each group (400-fold HE staining). NC: Normal control group, BLM: Bleomycin-induced systemic sclerosis model group, PESV-L: Low-dose PESV intervention group, PESV-M: Medium dose PESV intervention group, PESV-H: High-dose PESV intervention group, DXM: Dexamethasone intervention group.

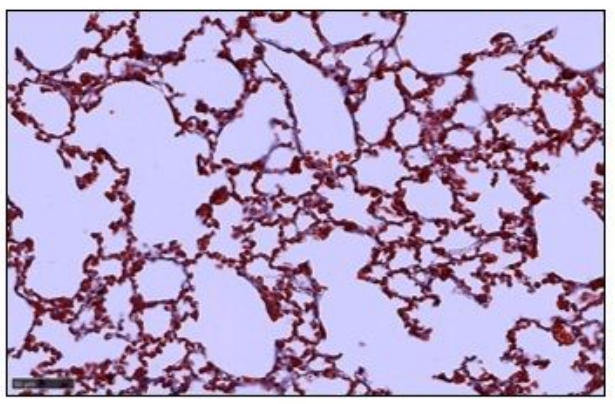

$\mathrm{NC}$

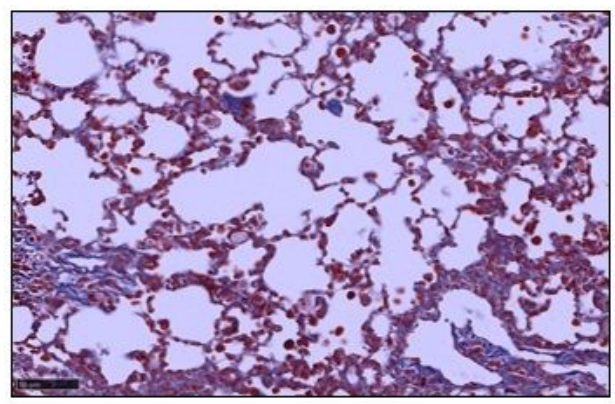

PESV-M

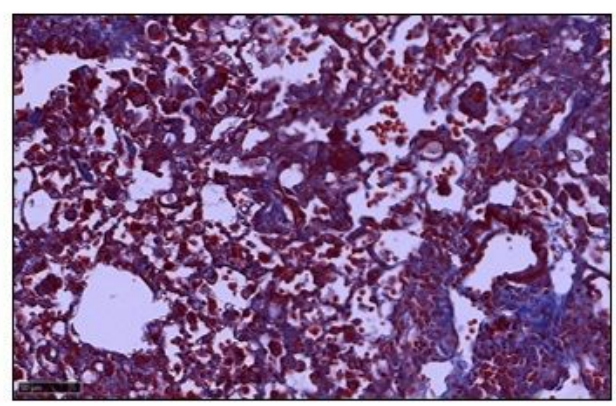

BLM

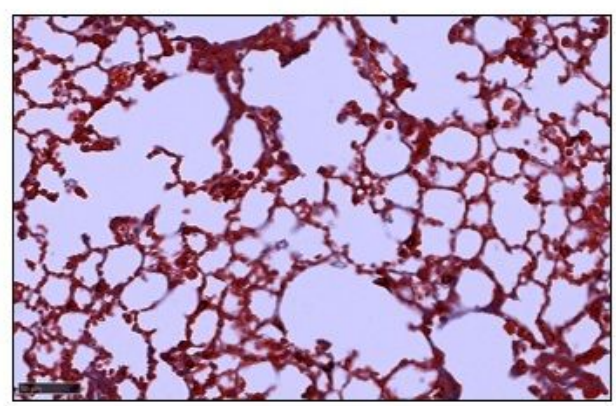

PESV-H

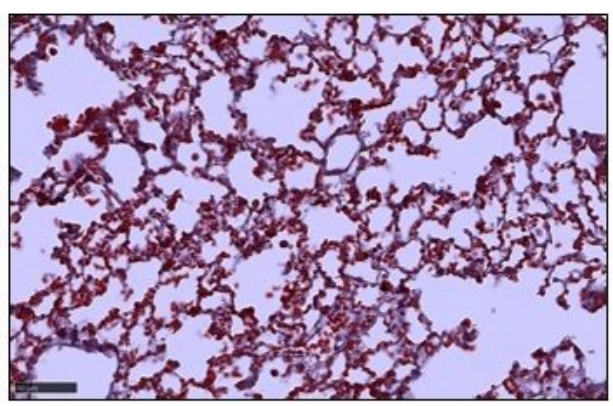

PESV-L

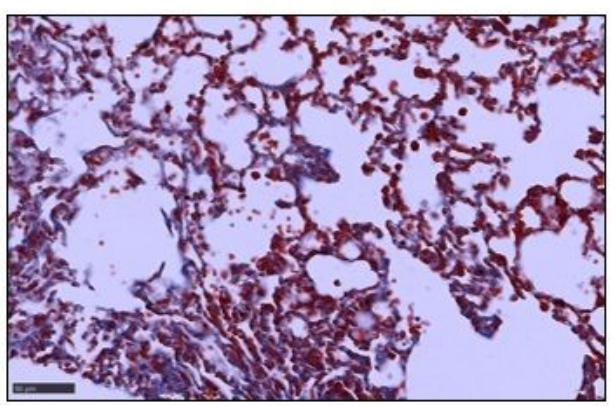

DXM 
Figure 2

Masson staining of lung tissues from mice in each group (400-fold). NC: normal control group, BLM: bleomycin-induced systemic sclerosis model group, PESV-L: low-dose polypeptide extract of PESV intervention group, PESV-M: Medium dose PESV intervention group, PESV-H: High-dose PESV intervention group, DXM: Dexamethasone intervention group.

A

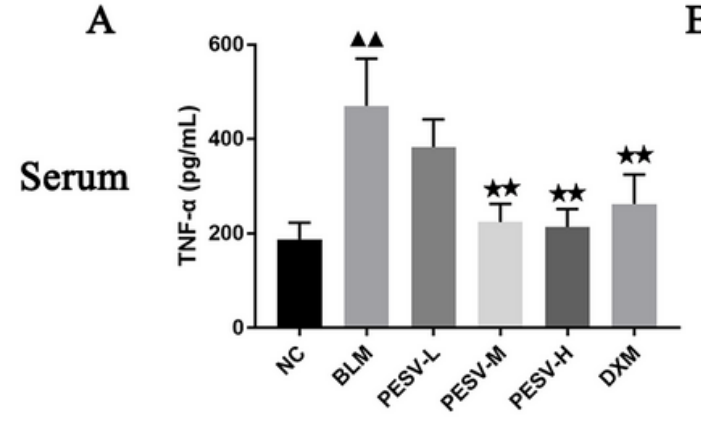

D

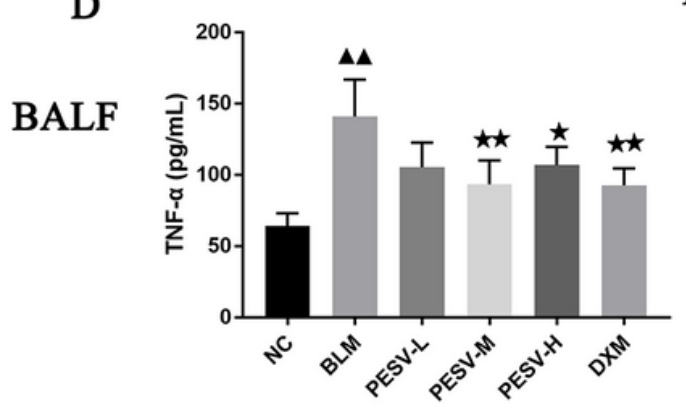

B

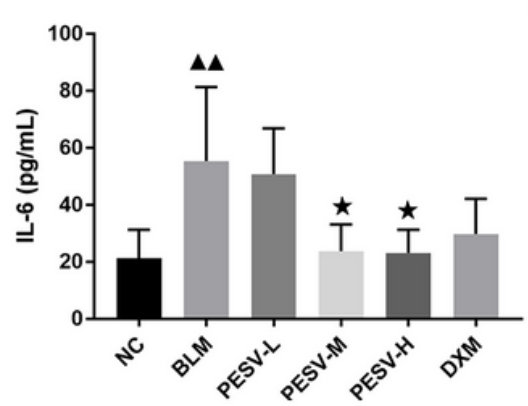

E

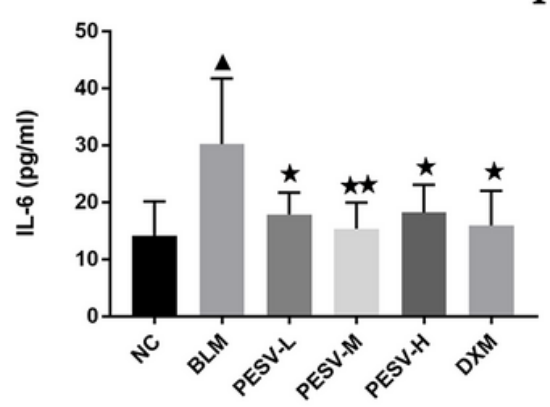

C

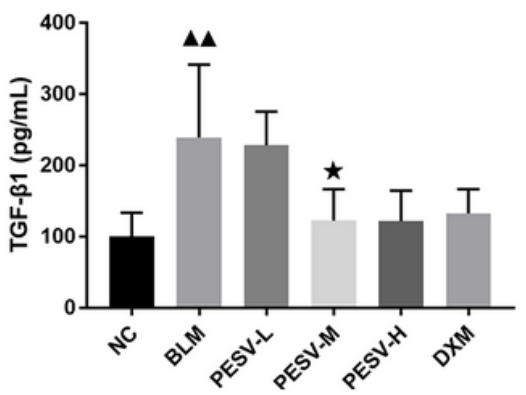

F

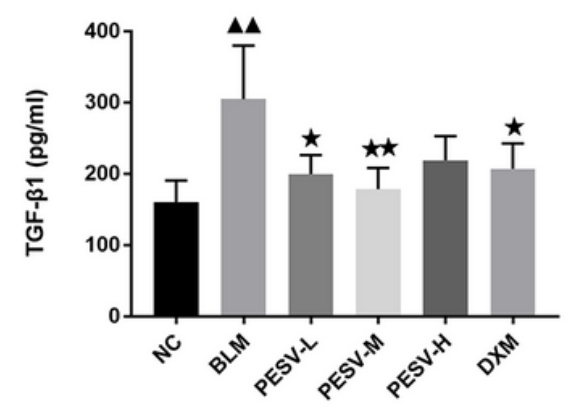

Figure 3

Contents of inflammatory cytokines TNF- $\alpha$, IL- 6 and TGF- $\beta 1$ in serum (A, B, C) and BALF (D, E, F) of mice in each group. NC: Normal control group, BLM: Bleomycin-induced systemic sclerosis model group, PESVL: Low-dose PESV intervention group, PESV-M: Medium dose PESV intervention group, PESV-H: Highdose PESV intervention group, DXM: Dexamethasone intervention group. Compared with the NC group, $P<0.05, \Delta \Delta P<0.01$; compared with the BLM group, $\otimes P<0.05$, $\otimes \otimes P<0.01$. Data represent the mean \pm $\mathrm{SEM}, \mathrm{n}=3$. 


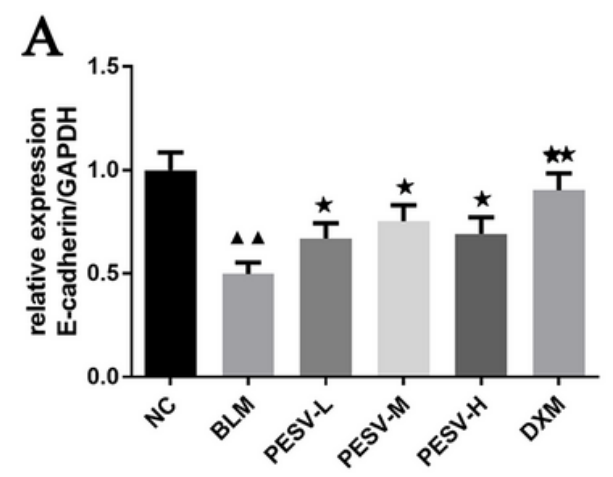

B

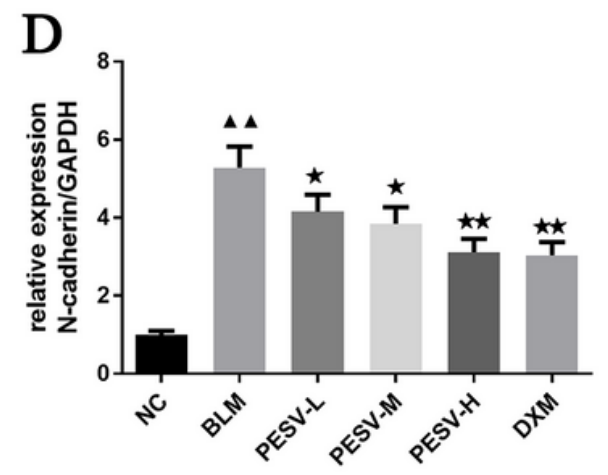

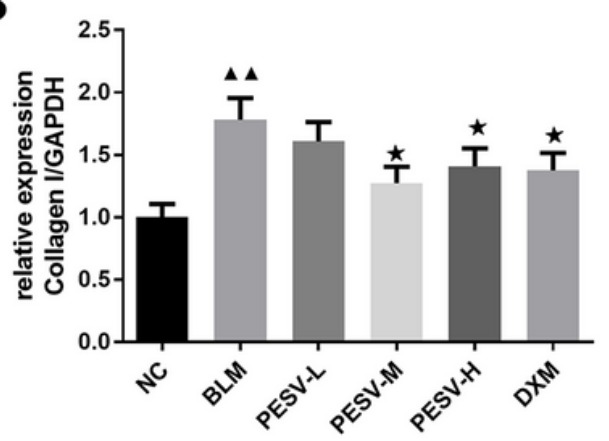

E

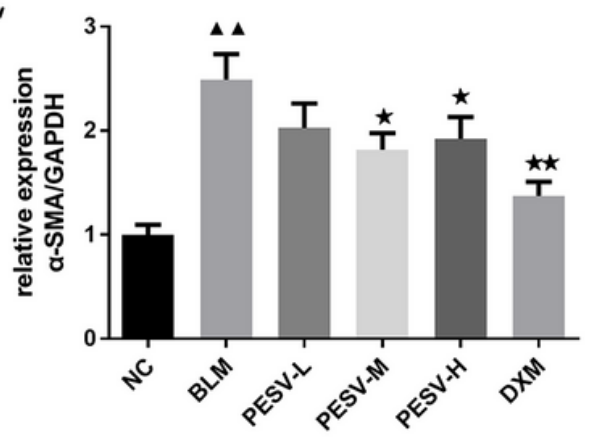

C

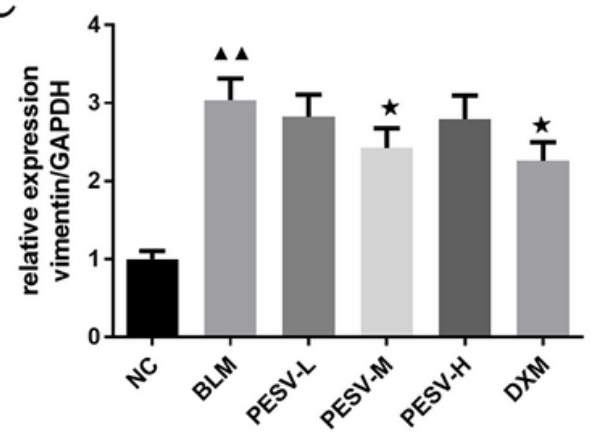

F

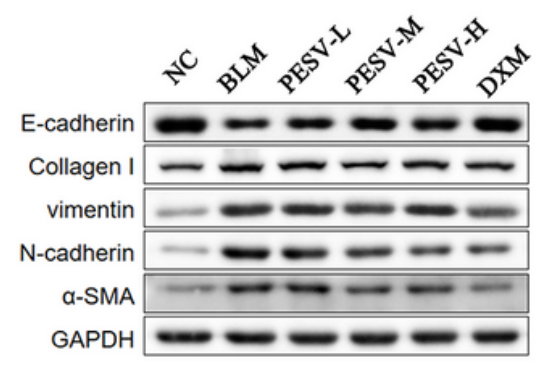

\section{Figure 4}

The expression levels of E-cadherin (A), collagenl (B), vimentin (C), N-cadherin (D), and a-SMA (E) proteins and their protein bands $(F)$ in the lung tissues of mice in each group. NC: Normal control group, BLM: Bleomycin-induced systemic sclerosis model group, PESV-L: Low-dose PESV intervention group, PESV-M: Medium dose PESV intervention group, PESV-H: High-dose PESV intervention group, DXM:

Dexamethasone intervention group. Compared with the NC group, $\boldsymbol{\Delta} P<0.05, \boldsymbol{\Delta} \boldsymbol{\Delta} P<0.01$; compared with the $B L M$ group, $\triangle P<0.05$, $\otimes \square P<0.01$. Data represent the mean $\pm S E M, n=3$. 


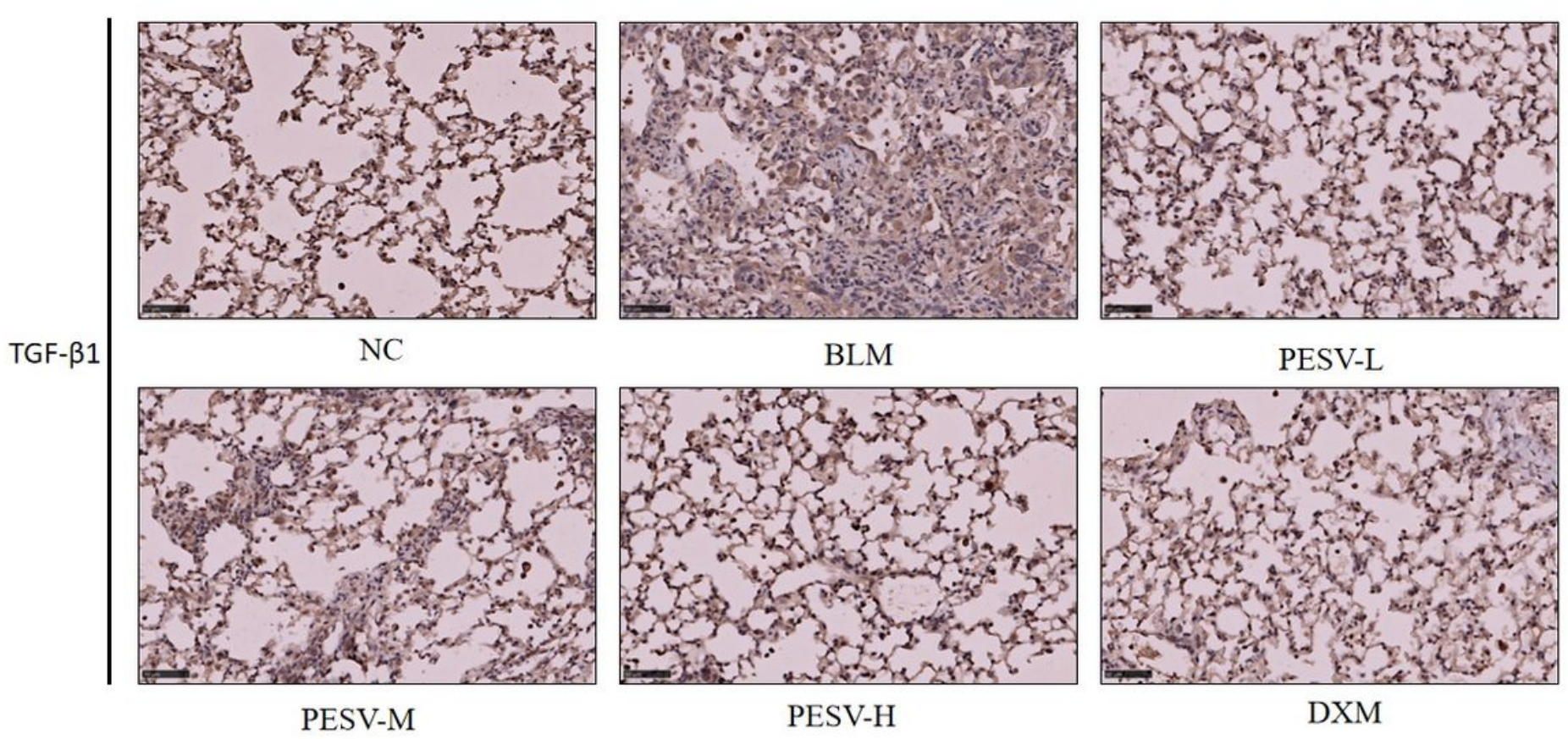

Figure 5

Immunohistochemical staining of TGF- $\beta 1$ in lung tissue of mice in each group (400-fold). NC: Normal control group, BLM: Bleomycin-induced systemic sclerosis model group, PESV-L: Low-dose scorpion venom intervention group, PESV-M: Medium dose PESV intervention group, PESV-H: High-dose PESV intervention group, DXM: Dexamethasone intervention group. 

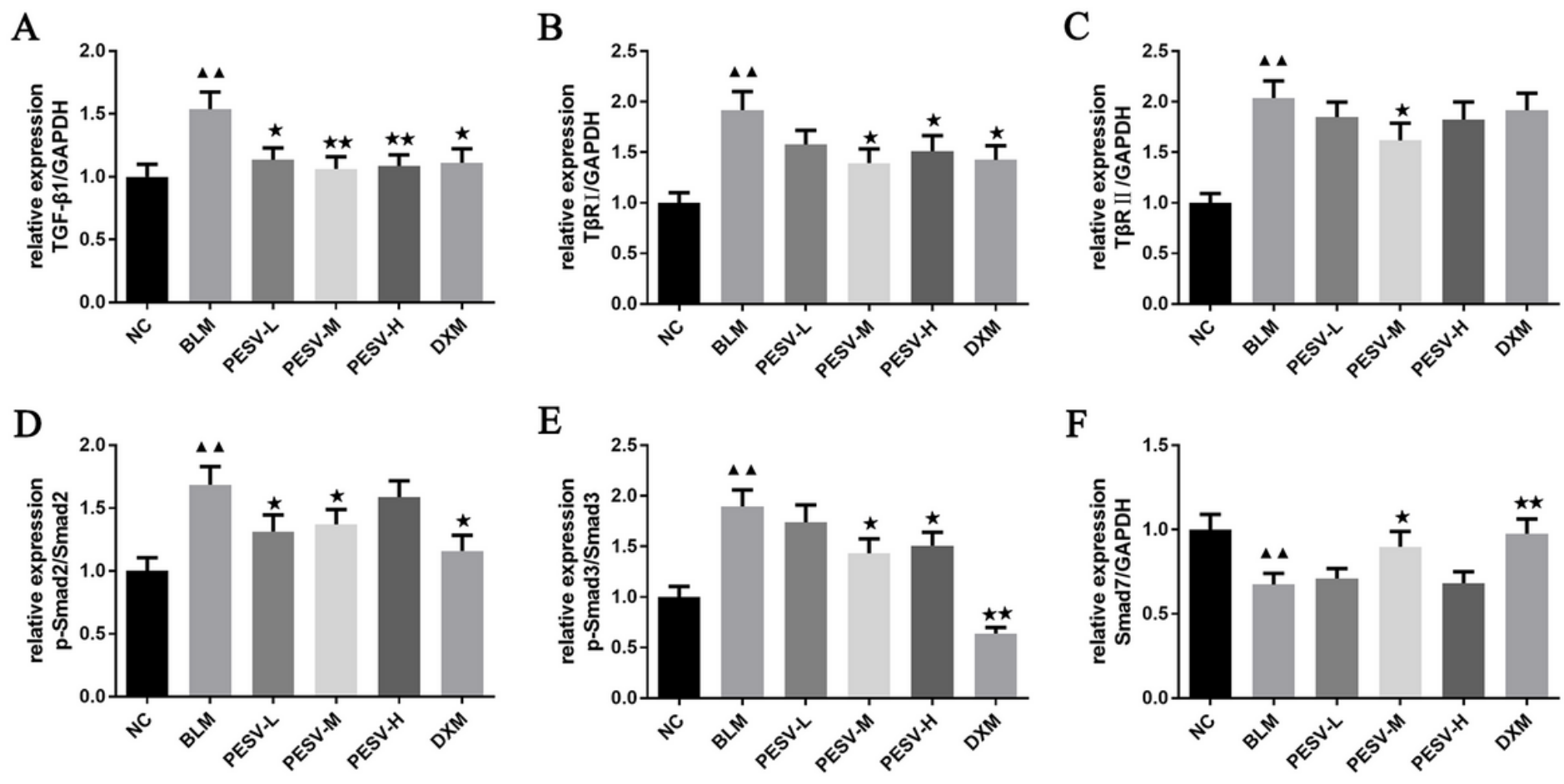

$\mathrm{E}$
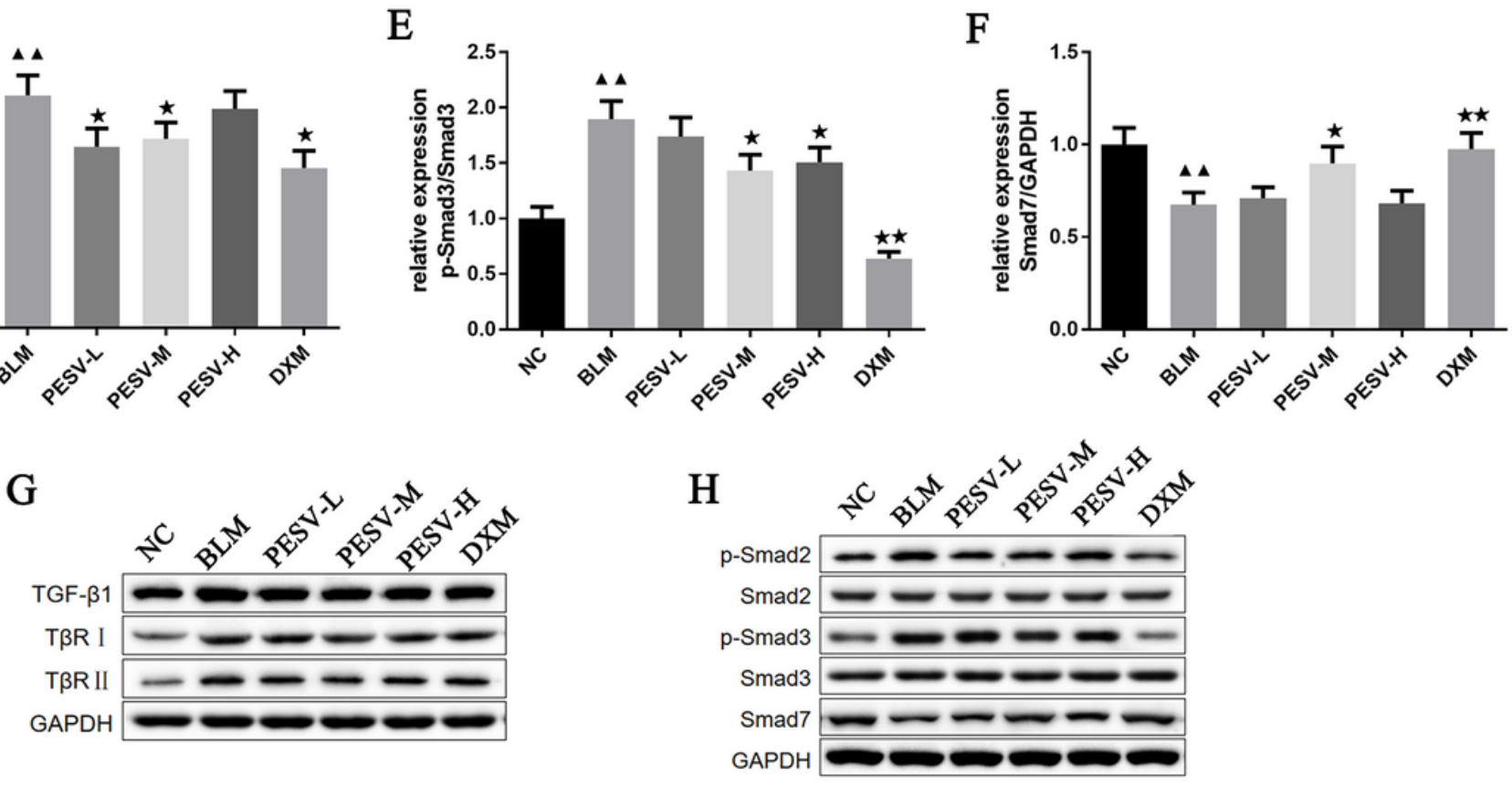

Figure 6

The expression levels of TGF- $\beta 1$ (A), T $\beta R I(B), T \beta R I I(C), p-S m a d 2$ (D), p-Smad3 (E), and Smad7 (F) proteins and their protein bands $(G, H)$ in the lung tissues of mice in each group. NC: Normal control group, BLM: Bleomycin-induced systemic sclerosis model group, PESV-L: Low-dose PESV intervention group, PESV-M: Medium dose PESV intervention group, PESV-H: High-dose PESV intervention group, DXM: Dexamethasone intervention group. Compared with the NC group, $\Delta P<0.05, \mathbf{\Delta} \Delta P<0.01$; compared with the BLM group, $\otimes P<0.05$, $ه \mathbb{P} P<0.01$. Data represent the mean $\pm S E M, n=6$.

\section{Supplementary Files}

This is a list of supplementary files associated with this preprint. Click to download.

- ContentsofinflammatorycytokinesIL6TNFandTGF1 inserumandBALF.xIsx

- Theoriginalblotwithchangesmarked.pdf

- Theoriginalblot.pdf 